\title{
Polyurethane and PTFE membranes for guided bone regeneration: Histopathological and ultrastructural evaluation
}

\author{
Adriana-Socorro-Ferreira Monteiro ${ }^{1}$, Luís-Guilherme-Scavone Macedo ${ }^{2}$, Nelson-Luiz Macedo ${ }^{3}$, Ivan Bal- \\ ducci $^{4}$
}

${ }^{1}$ DDS, MSc, PhD in Oral Pathology, Department of Biosciences and Oral Diagnostic, UNESP - São Paulo State University - São José dos Campos Dental School

${ }^{2}$ DDS, MSc, Pos-graduate, Restorative Dentistry, Department of Dental Materials and Prosthesis - UNESP São Paulo State University - São José dos Campos Dental School

${ }^{3}$ DDS, MSc, PhD, Assistant Professor, Department of Diagnosis and Surgery, Periodontics Division - UNESP São Paulo State University - São José dos Campos Dental School

${ }^{4}$ DDS, MSc, Assistant Professor, Department of Social Dentistry and Pediatric Clinics, Biostatistic Division - UNESP São Paulo State University - São José dos Campos Dental School

Correspondence:

Department of Diagnosis and Surgery

São José dos Campos Dental School, UNESP

Avenida Engenheiro Francisco José Longo, 777

Caixa Postal 314

CEP 12245-000 São José dos Campos, SP - Brazil

dri.monteiro@fosjc.unesp.br

\begin{abstract}
Monteiro AS, Macedo LG, Macedo NL, Balducci I.. Polyurethane and PTFE membranes for guided bone regeneration: Histopathological and ultrastructural evaluation. Med Oral Patol Oral Cir Bucal. 2010 Mar 1;15 (2):e401-6.

http://www.medicinaoral.com/medoralfree01/v15i2/medoralv15i2p401.pdf
\end{abstract}

Received: 13/02/2009

Accepted: 02/08/2009

\begin{abstract}
Objective: The purpose of this study was to research a membrane material for use in guided bone regeneration. Study design: In this study, 25 male Wistar rats were used to analyze the biocompatibility and degradation process of biomembranes. The morphological changes in subcutaneous implantations were assessed after 7, 14, 21, 28 and 70 days. The materials were made of polyurethane polymer (AUG) obtained from vegetal oil (Ricinus communis) and polytetrafluoroethylene membrane (PTFE). The surface characteristics of the physical barriers in scanning electronic microscopic (SEM) were also evaluated. Results: In both groups, the initial histological analysis showed moderate inflammatory infiltrate, which was predominantly polymorphonuclear. There was also a presence of edema, which was gradually replaced by granulation tissue, culminating in a fibrous capsule. In the AUG group, some multinucleated giant cells were present in the contact interface, with the space previously occupied by the material. However, membrane degradation was not observed during the period studied. According to the present SEM findings, porosity was not detected in the AUG or PTFE membranes. Conclusion: The researched material is biocompatible and the degradation process is extremely slow or may not even occur at all.
\end{abstract}

Key words: Guided tissue regeneration, bone regeneration, artificial membranes, bone substitutes, polytetrafluoroethylene, polyurethane. 


\section{Introduction}

Several surgical procedures to rebuild located deformities of the alveolar ridge were described in the prior literature. Such deformities can be originated from tooth extraction, mainly when it involves bone tissue removal. The reconstruction of bone defects is a complex process influenced by age, bone structure, vascularization, defect morphology and adjacent soft tissue $(1,2)$. Guided bone regeneration (GBR) was introduced to assist bone growth in order to restore the tissue in the treated area. Thereby, membranes are placed over bone defects to create a closed cavity and to act as a barrier preventing non-osteogenic cells from invading the bone defect (3). Prerequisites for an ideal barrier membrane include biocompatibility, cell occlusivity, tissue integration, spacemaking effect, and clinical manageability. In many experimental GBR studies, nonabsorbable membranes are used. Some disadvantages of such devices were mainly associated with the first generation of membranes made of e-PTFE: necessity of a second surgery to remove the membrane and early exposure of the material to the oral environment, allowing bacterial colonization and subsequent tissue infection (2). PTFE nonporous barrier has been successfully used in experimental and clinical studies. Since the material has no porosity, the attachment of bacterial cells to the surface of the membrane is hindered, allowing the exposition to the oral environment. Besides, PTFE material presents biocompatibility, low cost and easy handling (3).

A variety of absorbable membranes composed of polylactic acid, polygalactin 910, collagen and dura mater were introduced to overcome these problems. However, some membranes were associated with inflammatory reactions in the adjacent tissue or were quickly degraded by enzymatic activity of macrophages and neutrophils. Absorbable membranes that are available to the GBR are not able to keep appropriate space unless it has favorable defect morphology. Even if the membranes are initially able to keep the space, the material usually loses the strength and its implantation in the tissue. Only in situations where the bone defect margins maintain appropriately the membrane in position, favorable results have been reported. However, when bone defect does not support the physical barrier, bone regeneration failure occurs $(2,3)$.

The purpose of the present study is to investigate a new absorbable polyurethane-derived membrane (Ricinus communis) for using in GBR. The polymer was described as being biocompatible, osteoconductor, osteoinductor, antimicrobial, osseointegrable and absorbable (4-7), however, in the membrane form, few works were accomplished. The null hypothesis was that there would be no difference between the results obtained after membrane implantation in rats subcutaneous. The alternative hypothesis was that there would be a statistically significant difference.

\section{Material and Method}

\section{Microscopic analysis}

The biocompatibility of two types of membranes was evaluated by morphological alterations in subcutaneous of 25 male Wistar rats, weighting between 200 and $300 \mathrm{~g}$. This research was approved by the Committee of Ethics in Animal Research of São José dos Campos School of Dentistry - UNESP, according to the criteria of the animal protocol.

PTFE nonporous membrane (Tecnoflon-Brasflon, Ind. \& Com. Plasticos, São Paulo, SP), with $0.13 \mathrm{~mm}$ thickness, presents characteristics and biocompatibility appropriate to be applied in the GBR technique. The polyurethane barrier (AUG) (Augment-M ${ }^{\circledR}$, Ricinix Biomateriais. Poliquil, Belo Horizonte, MG), obtained by polymerization of the polyester polyol, derived from a tropical castor oil (Ricinus communis), with $0.13 \mathrm{~mm}$ thickness, was supplied in packages sterilized by gamma-radiation.

Preoperatively, the animals were weighed and sedated with analgesic-sedative and muscle-relaxing $(0.1 \mathrm{~mL} / 100 \mathrm{~g})$ Rompum (2\% aqueous solution of 2-(2,6xilidine)-5,6-dihydro-4H-1,3 thiazin, Bayer, Brazil) five minutes before administration of general anesthesia $(0.5 \mathrm{~mL} / 100 \mathrm{~g})$ Dopalen (ketamine hydrochloride, Agribrands do Brasil Ltda, Brazil).

After trichotomy and the asepsis of the dorsal region, two longitudinal incisions ( $2 \mathrm{~cm}$ extension) at a distance of $4 \mathrm{~cm}$ were performed in the medium line. The blunt dissection in the subcutaneous connective tissue was made through laterally with a round-tip scissor to material placement. The membranes were cut in disks of 1 $\mathrm{cm}$ diameter, and later implanted in the subcutaneous tissue. The polyurethane (AUG group) was inserted on the right side and the PTFE (PTFE group) was placed on the left side. The surgical wound was closed with 4-0 mononylon-interrupted sutures, for a first intention repair.

The animals were euthanased at 7,14,21,28 and 70 days with anesthetic overdose. The samples containing the material were immediately fixed in $10 \%$ formalin solution and routinely processed for histology. Longitudinal histological sections with $5 \mu \mathrm{m}$ thickness were stained with hematoxylin and eosin for analysis in light microscopy.

\section{Histomorphometric analysis}

Randomly, 3 of the 5 semiserial histological sections of each animal were evaluated by Axioscope 40 light microscope (Carl Zeiss, Germany) and Axiovision program (Carl Zeiss Vision Imaging System, Carl Zeiss, Germany). Histomorphometric analysis was performed to find out qualitative parameters of the inflammatory reaction. A blind study was conducted to measure the tissue outcome in the area using scores, observing the presence of neutrophils, lymphocytes, plasmocytes, 
eosinophils, macrophages. Based on these findings, the slides were then classified according to the following scores: 0 - Absence (when inflammatory cells were absent or found only within the blood vessels); 1 - Discrete (when inflammatory cells were sparsely present or in very small groups); 2 - Moderate (when inflammatory cells were densely present or in some groups); 3 - Intense (when inflammatory cells were found in the entire field or were present in a large number, configuring great severity).

After qualitative analysis and attribution of scores to the observed events, the slides were scored according to inflammation severity. A ranking scale was established, which ordered the slides in increasing degree of inflammatory reaction, ranging from absent to high inflammatory reaction. The data were submitted to Wilcoxon Signed Rank Test to allow the comparison among groups. Kruskal-Wallis nonparametric test was used to analyze the comparison among observation periods ( $5 \%$ significance).

\section{Scanning electronic microscopy}

The surface characteristic of the polyurethane and PTFE membranes was evaluated in SEM. The samples were fastened in an aluminum stub and covered with gold, using a sputtering evaporation process, through Desk II (Denton Vacuum), for materials metallization, ensuring good resolution for detecting the characteristics of the membrane surface. After processing, samples were observed in an SEM (JMS 5310-JEOL) at magnification of $35 \mathrm{X}, 500 \mathrm{X}, 2000 \mathrm{X}, 7500 \mathrm{X}$ using $15 \mathrm{kV}$, and photographed in a digital camera system coupled to SEM.

\section{Results}

\section{Histological analysis}

The histological analysis showed in both groups a moderated initial inflammatory infiltrate with polymorphonuclear predominance, edema and granulation tissue (Fig. 1). The inflammatory infiltration was gradually substituted by granulation tissue, which then turned into a fibrous capsule formation. In AUG group some multinucleated giant cells were present in the contact interface, over the area previously occupied by the material. However, membrane degradation was not observed in the studied period (Fig. 2).

\section{Histomorphometric analysis}

No statistically significant differences were found in the analysis of the inflammatory response when comparing the tested materials (all $p$ values were over 0.05 ). $\mathrm{Ne}$ vertheless, when testing the influence of the time on the degree of inflammation, the inflammatory process decreased in a longer experimental interval (Table 1).

Scanning electronic microscopy

The morphological characterization of the membrane surface was determined with scanning electronic microscopy (SEM). The images from polyurethane barrier (AUG) showed plane and homogeneous surface saliencies and depressions, and an irregular outline as well. Besides, granular structures of several sizes were shown, and found mainly in the areas among the saliencies. In the images obtained from PTFE barrier, a plane and regular surface was observed. No porosity was detected in the images of the two types of membranes (Fig. 3).
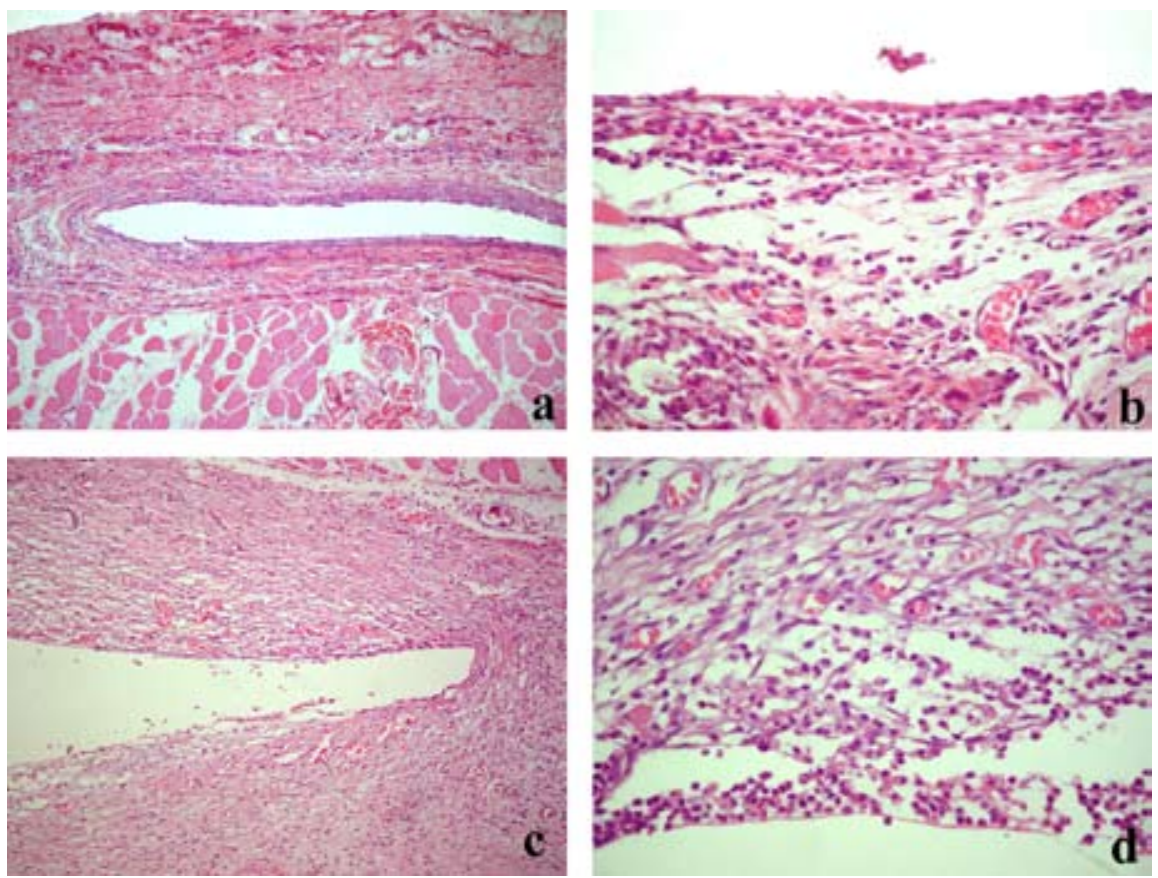

Fig. 1. a) AUG 7 days: granulation tissue with inflammatory infiltrate around the material. HE, 100x; b) AUG 7 days: mono and polymorphonuclear inflammatory infiltrate. HE, 400x; c) PTFE 7 days: granulation tissue with inflammatory infiltrate and edema round the material. HE, 100x; d) PTFE 7 days: mono and polymorphonuclear inflammatory infiltrate. HE, 400x. 

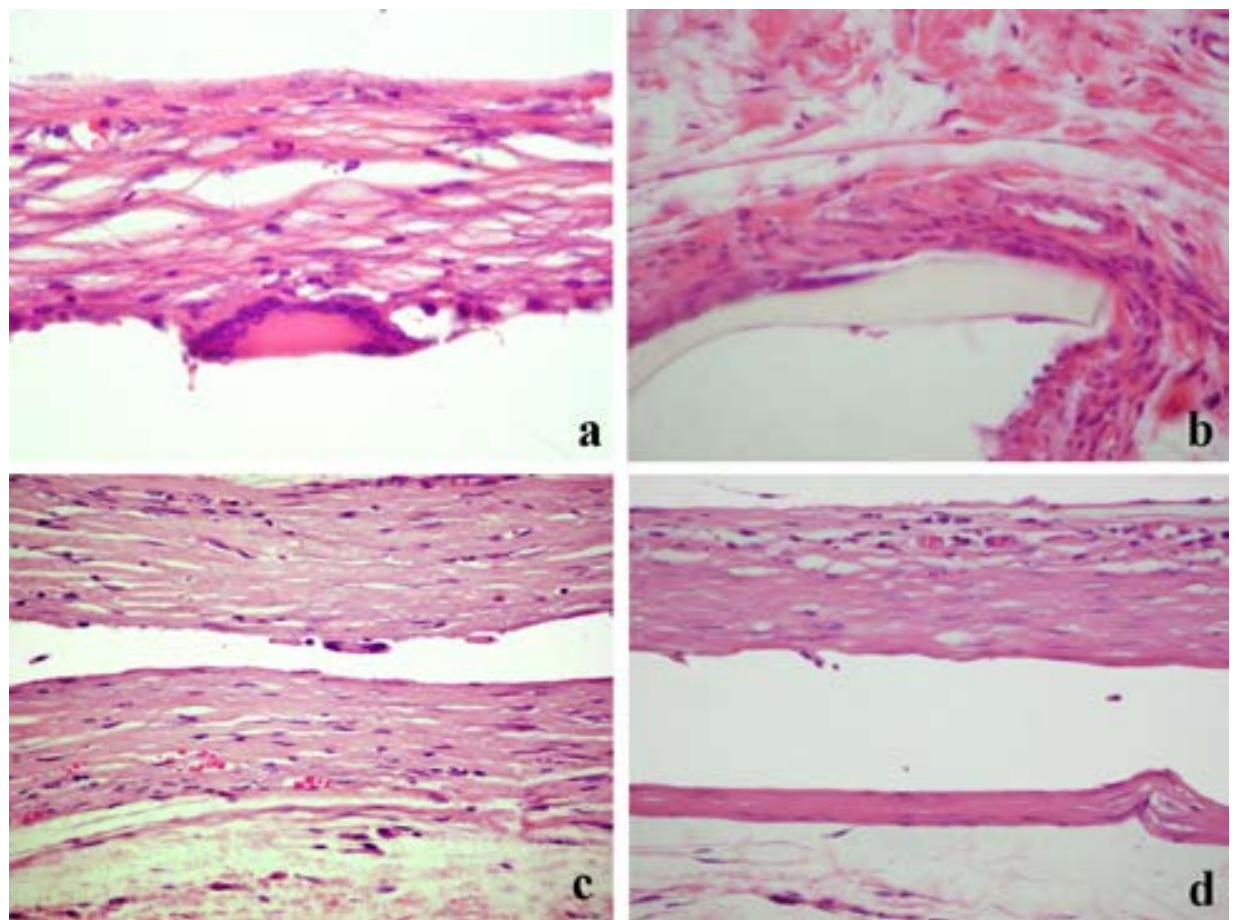

Fig. 2. a) AUG 14 days: multinucleated giant cells in the fibrous capsule. HE, 630x; b) AUG 28 days: polyurethane membrane - surrounded by a capsule of cellular connective tissue. HE, 400x; c) AUG 70 days: dense fibrous capsule. HE, 400x; d) PTFE 70 - days: dense fibrous capsule. HE, 400x.

Table 1. Comparison between treatments: AUG versus PTFE. Distribution values data (modal score) arou median value (dot plot) by period (d: days).
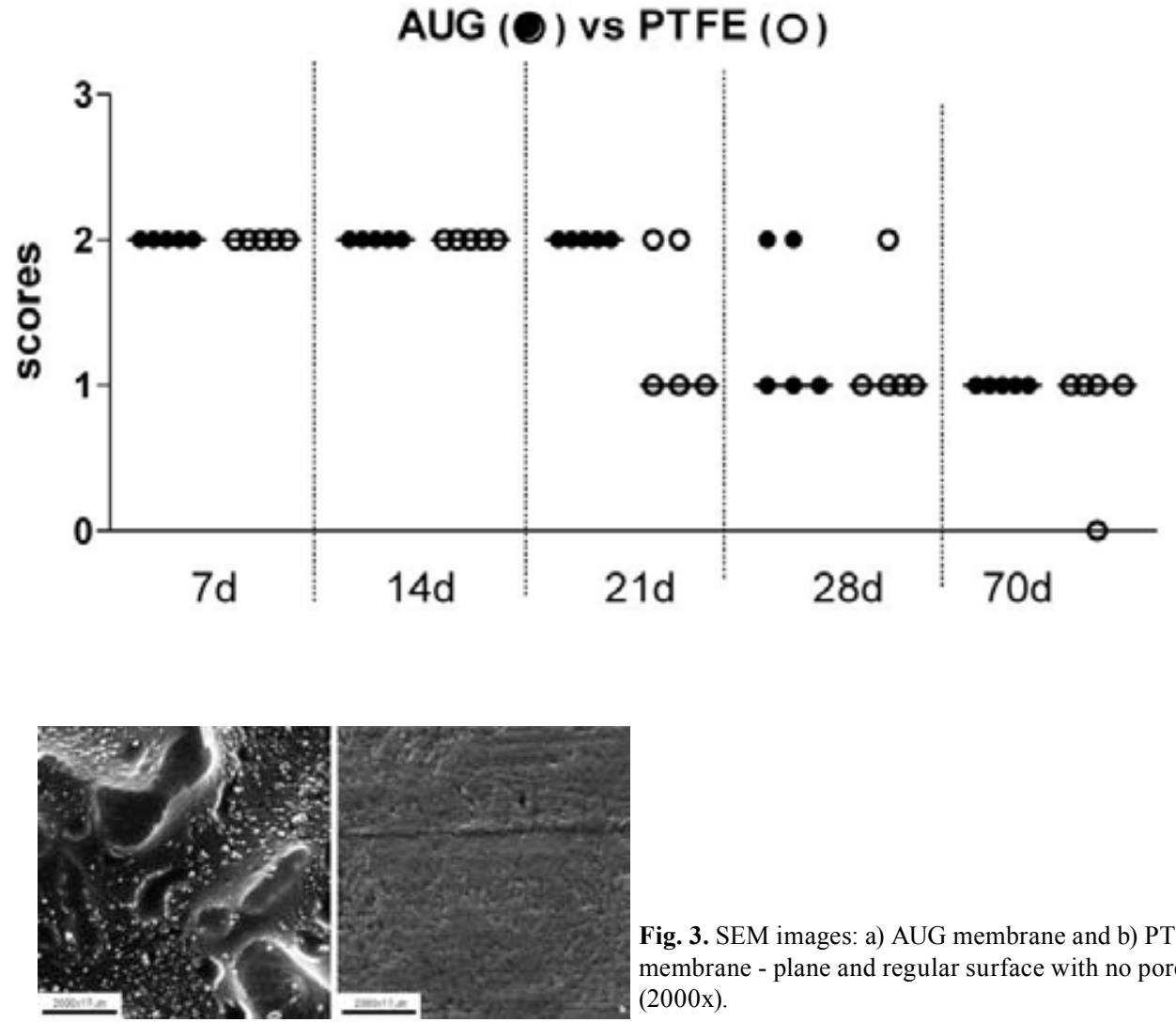

Fig. 3. SEM images: a) AUG membrane and b) PTFE membrane - plane and regular surface with no pores (2000x). 


\section{Discussion}

Although the GBR literature is vast and exhaustive, there are several common themes that still need to be researched and discussed. In fact, an ideal membrane does not exist. The PTFE physical barriers are preferable because this material is associated to a growth of bone tissue with larger and higher quality. On the other hand, the absorbable membrane degradation may resul in the development of local inflammatory process, causing a small amount of bone formation (2).

Non-absorbable barriers require a second surgical procedure for removal. The need for additional surgery is accompanied by concerns over patient acceptance, time, cost, and possible morbidity associated with any surgical procedure. However, some absorbable membranes can fail in keeping the space or not remaining long enough to allow bone growth. Therefore, longevity and space-maintaining ability are the most challenging concerns regarding absorbable membranes in GBR (1-3).

Bioabsorbable membranes are composed of a wide variety of materials including collagen, polylactic acid, polyglactin-910, acellular dermal matrix, dura mater, chitosan, periosteum, and calcium sulfate. The most common material is collagen, which can be modified through various collagen cross-linking processing techniques to vary the resorption rate. Because of alveolar bone and periodontal ligament containing collagen, using a collagen membrane might impart some additional advantages for GBR purposes by augmenting its native properties. Collagen facilitates hemostasis and therefore, wound stability by promoting platelet aggregation in addition to promoting fibroblast migration, which could accelerate wound closure (8). However, the collagen membranes have unsatisfactory mechanical properties, as they are not stiff enough to resist soft-tissue pressure during healing. The use of stiffer membranes, such as the polyurethane material, in GBR could solve the problem of membrane collapse and could improve the restoration of bone defects in maxillofacial surgery. The porous physical barriers allows the passage of fluids and nutrients, creating a suitable environment for osteogenesis, soft tissue integration and anchorage, avoiding the membrane exposure to the oral cavity $(2,9)$. According to these authors, the satisfactory results were attributed to the membrane porosity, since the nonporous barrier reduces or interrupts the blood supply.

The pores of the membrane exposed to the oral cavity allows the colonization of microorganisms causing local infection, reducing the amount of bone tissue, and should be removed as soon as possible $(2,10)$. Nowadays, there is no treatment to extend the permanence of the exposed membrane; the elimination of microorganisms by local chlorhexidine applications or antibiotics use is not effective. According to the present SEM findings, porosity was not detected in the AUG or PTFE membranes. Dense barriers present important advantages about the retention of bacteria in comparison to porous membranes. Besides, the permeability and integration of the membrane are not required for the new bone formation in GBR technique $(3,11)$.

The minimum biological condition for the long term success of implanted materials is biocompatibility. Any material inserted into the tissue causes an immunological response, and the biocompatibility is determined according to the extension, intensity and duration of the reaction. In this study, the tissue response after implantation of two types of membranes was evaluated in subcutaneous of rats. The biocompatibility of PTFE was corroborated in this study (3). Probably, the smooth surface quality, nonporosity and the material chemical purity have contributed to the good results obtained. Besides the biocompatibility, the use of PTFE as a physical barrier is based on the maintenance of space for blood clot formation. The PTFE barriers have enough firmness against the soft tissue pressure, an important requirement for bone regeneration.

The castor oil polyurethanes present an excellent biological compatibility (12-14). The observations on the inflammatory infiltrate in the samples treated with polyurethane barrier, showed an initial inflammatory reaction which decreased along the time (4), and the presence of inflammatory multinuclear giant cells (IMGC) (7). Although the tissue reaction varied from mild to absent, the polymer acts as a strange body, promoting an inflammatory infiltrate of mononuclear prevalence that, on average, was not significant (13). In this study, the fibrous connective tissue capsule, outlining the space previously occupied by the material was permeated with IMGC and inflammatory mononuclear cells.

A composite of (Ricinus communis) polyurethane (RCP) and alkaline phosphatase (ALP) incubated in synthetic body fluid (SBF) were analyzed by the response of osteoblastic cells (15). The composite RCP+ALP revealed to be cytotoxic and the authors justified that SBF incubation could be a useful alternative to improve the biological properties of the RCP. In the present study, necrosis areas and cellular changes were not observed, as hydropic and hyaline degeneration, and triglycerides and cholesterol collection, which would be cytotoxicity signs.

The antimicrobial action of polyurethane was shown in an in vitro study using the castor oil plant detergent (16). Despite the results of that study have demonstrated action only against Gram-positive microorganisms, microbial decrease was reported in root channel in an in vitro work performed after application of the polyurethane detergent (5). This reduction could be justified because of the detergent action on Gram-positive microbiota, interfering in the bacterial environment that causes the biofilm dissolution. Possibly the porosity absence, as 
demonstrated in SEM, could be an important factor to decreasing rate of bacterial growth in clinical situation. In the present work, the barriers exposition was not observed, as well as no infection.

The studies with polymer of polyurethane as a bone graft substitute had demonstrated contradictory and questionable results on the material persistence. Some authors believe that the degradation products of the polymer are nontoxic to cells. Foreign-body giant cells did accumulate around the polymer and in its pores, suggesting the degradation is facilitated by hydrolysis as well as by giant cells. More important, subcutaneous implants of the polymer allowed infiltration of vascular and connective tissue, suggesting the free flow of fluids and nutrients in the implants $(7,14)$.

The osteogenic tissue presence in the polymer surface and the membrane incorporation in the newly formed bone tissue were related $(4,17)$. Following the insertion of the polyurethane resin into the alveolar bone of dogs, it was observed that the material was replaced by osteoid and bone tissues (12). Implantation of flakes of castor oil resin in rat dental alveolus showed a close contact with the newly formed bone in some regions, as the relative volume of bone trabeculae increased. On the other hand, some authors noted a capsule formed by fibrous connective tissue involving the polymer $(18,19)$. In the present study, implanting the polymer in the subcutaneous layer of the skin, osteogenic tissue formation was not observed, questioning the osteoinduction activity of the material.

Despite the evidences of polyurethane absorbing properties in previous research $(19,20)$, AUG absorption were not observed during all the periods in the present work and others $(13,17)$. In many clinical situations a resorption time not extending beyond 6-12 months is mandatory in order not to lose the advantages of resorbability (2).

The relevant properties of the polyurethane membrane observed in this study, can be important for a successful treatment outcome with GBR procedures. Thus, the positive results and successful perspectives application in the Dentistry could bring advantages to clinical practice in the treatment of bone defects. However, accurate researches utilizing specific methods to prove other properties and the explanation for the behavior of the polyurethane membrane are required. Also, when used in reconstructive surgeries, the effects of the polyurethane physical barrier in different tissues could complement the results of the present investigation.

\section{Conclusions}

Based on the obtained results, it can be concluded that polyurethane membrane is a nonporous, biocompatible, well tolerated by the organism, and that degradation process is extremely slow or perhaps may not occur at all.

\section{References}

1. Jung RE, Zwahlen R, Weber FE, Molenberg A, Van Lenthe GH, Hammerle $\mathrm{CH}$. Evaluation of an in situ formed synthetic hydrogel as a biodegradable membrane for guided bone regeneration. Clin Oral Implants Res. 2006;17:426-33.

2. Hämmerle $\mathrm{CH}$, Jung RE. Bone augmentation by means of barrier membranes. Periodontol 2000. 2003;33:36-53.

3. De Macedo NL, De Macedo LG, Monteiro Ado S. Calcium sulfate and PTFE nonporous barrier for regeneration of experimental bone defects. Med Oral Patol Oral Cir Bucal. 2008;13:E375-9.

4. Carvalho TL, Araújo CA, Teófilo JM, Brentegani LG. Histologic and histometric evaluation of rat alveolar wound healing around polyurethane resin implants. Int J Oral Maxillofac Surg. 1997;26:149-52. 5. Ferreira CM, Da Silva Rosa OP, Torres SA, Ferreira FB, Bernardinelli N. Activity of endodontic antibacterial agents against selected anaerobic bacteria. Braz Dent J. 2002;13:118-22 .

6. Zhang JY, Beckman EJ, Piesco NP, Agarwal S. A new peptidebased urethane polymer: synthesis, biodegradation, and potential to support cell growth in vitro. Biomaterials. 2000;21:1247-58.

7. Leite FR, Ramalho LT. Bone regeneration after demineralized bone matrix and castor oil (Ricinus communis) polyurethane implantation. J Appl Oral Sci. 2008;16:122-6.

8. Bashutski JD, Wang HL. Periodontal and endodontic regeneration. J Endod. 2009;35:321-8.

9. Jovanovic SA, Hunt DR, Bernard GW, Spiekermann H, Wozney JM, Wikesjö UM. Bone reconstruction following implantation of rhBMP-2 and guided bone regeneration in canine alveolar ridge defects. Clin Oral Implants Res. 2007;18:224-30.

10. Li J, Wang HL. Common implant-related advanced bone grafting complications: classification, etiology, and management. Implant Dent. 2008;17:389-401.

11. Schmid J, Hämmerle CH, Olah AJ, Lang NP. Membrane permeability is unnecessary for guided generation of new bone. An experimental study in the rabbit. Clin Oral Implants Res. 1994;5:125-30.

12. König Júnior B, Forger SE, Mascaro MB, Beck TJ. Biocompatibility of the polyurethane resin of the castor bean inserted into the alveolar bone of the dog. Ann Anat. 1999;181:581-4.

13. Boëck-Neto RJ, Gabrielli MF, Shibli JA, Marcantonio E, Lia RC, Marcantonio E Jr. Histomorphometric evaluation of human sinus floor augmentation healing responses to placement of calcium phosphate or Ricinus communis polymer associated with autogenous bone. Clin Implant Dent Relat Res. 2005;7:181-8.

14. Ganta SR, Piesco NP, Long P, Gassner R, Motta LF, Papworth $\mathrm{GD}$, et al. Vascularization and tissue infiltration of a biodegradable polyurethane matrix. J Biomed Mater Res A. 2003;64:242-8.

15. Beloti MM, De Oliveira PT, Tagliani MM, Rosa AL. Bone cell responses to the composite of Ricinus communis polyurethane and alkaline phosphatase. J Biomed Mater Res A. 2008;84:435-41.

16. Leonardo MR, Da Silva LA, Filho MT, Bonifácio KC, Ito IY. In vitro evaluation of the antimicrobial activity of a castor oil-based irrigant. J Endod. 2001;27:717-9.

17. Pereira-Júnior OC, Rahal SC, Iamaguti P, Felisbino SL, Pavan PT, Vulcano LC. Comparison between polyurethanes containing castor oil (soft segment) and cancellous bone autograft in the treatment of segmental bone defect induced in rabbits. J Biomater Appl. 2007;21:283-97.

18. Mendonça JC, De Rossi R, Inouye CM, Bazan DR, Monteiro JC, Mendonça JP. Morphology of autogenous bone graft and castor oil polyurethane in the infraorbital rim of rabbits: a comparative study. Acta Cir Bras. 2006;21:341-7.

19. Gogolewski S, Gorna K. Biodegradable polyurethane cancellous bone graft substitutes in the treatment of iliac crest defects. J Biomed Mater Res A. 2007;80:94-101.

20. Laureano Filho JR, Castelo Branco Bde L, Andrade ES, Barbosa JR. Histological comparison of demineralized bone matrix and the Ricinus communis polymer on bone regeneration. Braz J Otorhinolaryngol. 2007;73:186-92. 